\title{
Dietary Addition of Astragalus Fermented by Lactobacillus Plantarum Improved Laying Performance, Egg Quality, Antioxidant and Immunological Status and Intestinal Microbiota in Laying Hens
}

\section{Hongtao Shi}

Henan University of Animal Husbandry and Economy Library

\section{Baiyu Wang}

Henan Agricultural University

Chuanzhou Bian

Henan University of Animal Husbandry and Economy

Yingqian Han ( $\nabla$ twgjl@163.com )

Henan Agricultural University

Hongxing Qiao

Henan University of Animal Husbandry and Economy

\section{Research}

Keywords: fermented Astragalus, laying hens, egg quality, antioxidant and immunological status, 16S rRNA, intestinal microbiota

Posted Date: May 22nd, 2020

DOl: https://doi.org/10.21203/rs.3.rs-27804/v1

License: (c) (1) This work is licensed under a Creative Commons Attribution 4.0 International License. Read Full License 


\section{Abstract \\ Background}

In the era of increased antibiotic resistance and ever stricter control on antibiotic use, it is urgent to develop green, safe and non-residue alternatives to antibiotics applied to the poultry industry. To this end, we supplied the potential Lactobacillus Plantarum (L. Plantarum) fermented Astragalus in the diet of laying hens, with a final addition of $3 \%$. Its effects have been assessed on laying performance, egg quality, antioxidant and immunological status and intestinal microbiota, and are compared to the control group, to the Astragalus group containing 3\%o unfermented Astragalus, and to the L. Plantarum group containing $2 \%$ L. Plantarum $\left(1 \times 10^{8} \mathrm{CFU} / \mathrm{mL}\right)$.

\section{Results}

During the second half of the experimental period (15 to 28 days), the egg production rate was significantly higher in the fermented Astragalus group than that in the other groups, with the fermented Astragalus group having the lowest feed conversion ratio. No significant difference $(P>0.05)$ was observed among treatments on egg quality. Fermented Astragalus-treated hens exhibited significantly increased catalase (CAT), glutathione peroxidase (GSH-Px), superoxide dismutase (SOD) and total antioxidant capacity (T-AOC) in serum, and reduced malondialdehyde (MDA) in serum. Furthermore, fermented Astragalus supplementation resulted in a significant increase in ileal microbiota abundance relative to control.

\section{Conclusions}

Feeding laying hens with L. Plantarum fermented Astragalus has beneficial effects on production, antioxidant potential, immunity and ileal microbiota. L. Plantarum fermented Astragalus is expected to be a novel feed additive used in poultry production.

\section{Background}

Eggs are one of the most crucial sources of animal protein and nutritional content in human diets. Due to the widespread use of antibiotics in poultry, drug residues in eggs have been gaining worldwide concern in the past few years [1]. In addition, antibiotic abuse has led to intestinal dysbacteriosis, diarrhea, immunocompromised state [2]. Thus, it is urgent to develop green, safe and non-residue alternatives to antibiotics applied to the poultry industry. Traditional Chinese herbal medicines are the gem of China with characteristics of safety, efficiency, and low residue and are widely used in preventive or therapeutic strategies for animal diseases [3]. Traditional Chinese herbal medicines have been used as feed additives for growth promotion and improvement of immunity and various effects, including anti-bacterial, anti-viral and antioxidative activities $[4,5]$. Since ancient times, traditional Chinese herbal medicines can be processed by microbial fermentation for improving its quality [6]. For example, fermentation of Chinese herbal medicine mediated by microbes can degrade macromolecule-materials into small ones and reduce 
their side effects [7]. Because microorganisms and their metabolic products can regulate the bioactive products of traditional Chinese herbal medicines, there is a close relationship between microorganisms and traditional Chinese herbal medicines.

Astragalus is a universal traditional Chinese herbal medicine and its main active pharmaceutical ingredients include polysaccharides, saponins, flavonoids, anthraquinones, alkaloids, amino acids, $\beta$-sitosterol and metallic elements [8]. Astragalus has been reported to possess anti-inflammatory [9], anti-viral [10] and antioxidant [11] activities and to enhance immunity [12], and it has been widely used in livestock. Nevertheless, challenges to the extraction yield of Astragalus functional ingredients are raised due to the recalcitrance of plant cell walls, and novel strategies for the improvement of Astragalus utilization efficiency need to be focused. The trend of microbial fermentation offers the possibility for addressing the above problem. In recent years, a research revealed that utilizing the fungus Aspergillus to ferment the Astragalus can significantly increase its phenolic contents and antioxidant activity, and the solid-state bioprocessing strategy could be an innovative approach to enhance the antioxidant activity of Astragalus [13]. Our previous studies have confirmed that the solid fermentation of Astragalus by L. Plantarum promotes the extraction yield of Astragalus active components and the production yield of organic acids [14]. Further investigation showed that fermented Astragalus improves broiler growth performance, enhances serum antioxidant status, and reduces fecal pathogenic microbiota of broiler chickens [15].

Over the last few years, there has meant considerable research on the application of Astragalus polysaccharide as feed additive in livestock including laying hens. However, there has not been a systematic appraisal of the application of Astragalus fermented by L. Plantarum as feed additive in laying hens. In this study, we investigated the possible effects of Astragalus fermented by L. Plantarum on egg production, egg quality, antioxidant status, immune factors expression and gut microbiome of laying hens, combining the classical culture and detection methods with high throughput sequencing.

\section{Methods}

\section{Fermentation of Astragalus}

L. plantarum (CGMCC 1.557) was purchased from the China General Microbiological Culture Collection Center (CGMCC) (Beijing, China). Te dried root of Astragalus membranaceus (Fisch.) Bge. var. mongholicus was obtained from Gansu Huisen Pharmaceutical Development Co., Ltd. (Minxian, Gansu, China) and verified by Dr. JingYu Zhang (Henan University of Traditional Chinese Medicine, Zhengzhou, Henan, China). The purchased Astragalus was crushed into powder and filtered with a 100-mesh filter for further studies. The fermentation of Astragalus was performed following the method reported in our previous publications with slight modification (Qiao et al 2018b). Briefly, dried Astragalus powder (7,500 g) was inoculated with $L$. plantarum $\left(1 \times 10^{6}\right.$ colony forming unit (CFU) per gram) with a water content of $45 \%$, and Astralagus-L. plantarum mixtures were aliquot into $35 \times 45-\mathrm{mm}$ plastic film bags. The bags were sealed for fermentation at $37^{\circ} \mathrm{C}$ for 30 days, and then dried out at room temperature for future use. 


\section{Experimental Design, Diets and Management}

Two hundred and forty healthy Hy-Line Gray hens (351 days, Zhengzhou, China) were acclimated with the basal diets for 7 days. Then, hens were randomly divided into 4 groups (fermented Astragalus group, Astragalus group, L. plantarum group and control group), each containing five replicates, with 12 hens per replicate. The control group was fed with the basal diet; the L. plantarum group was fed with the basal diet supplemented with $2 \%$ Lactobacillus solution $\left(5 \times 10^{8} \mathrm{CFU} / \mathrm{mL}\right)$ through uniform spraying; the Astragalus group was fed with the basal diet supplemented with 3\%o Astragalus, and fermented Astragalus group was fed with the basal diet supplemented with 3\%o fermented Astragalus (pre-experimental results showed that supplementing at a rate of $3 \%$ of diet achieves optimal results). The trial lasted for 35 days (7-day adaptation period and 28-day experimental stage). The hens were housed in a clean environment with good ventilation and artificial lighting allowed $16 \mathrm{~h}$ of lighting per day, and with water and food ad libitum. The basal diet of all groups was the same and prepared according to the NRC (1994) laying hen nutrition requirement standard. The composition and nutrient levels of basal diet were showed in Table 1. All animal experiments were conducted according to the Guidelines for the Care and Use of Experimental Animals established and approved by the Laboratory Animal Management Committee of Henan University of Animal Husbandry and Economy (HNMY 1606).

\section{Hen Productivity and Egg Quality}

During the experimental period, egg production, broken egg production, egg weight and feed intake were recorded daily. The egg production rate and the feed conversion ratio (FCR) (feed intake/egg weight gain) during day 1 to day 14 and day 15 to day 28 were calculated to assess the laying performance. On day 14 and day 28 , five eggs from each replicate were randomly sampled and measured egg quality parameters of egg shape index (ESI), eggshell strength (ESS), eggshell thickness (EST), albumen height (AH), haugh unit $(\mathrm{HU})$, yolk color (YC) and yolk weight (YW).

\section{Serum Antioxidant Indices}

On day 14 and day 28 , one hen from each replicate was randomly selected. Following blood collection from heart, the serum was isolated and stored at $-20^{\circ} \mathrm{C}$ until use. The CAT assay kit, GSH-Px assay kit, SOD assay kit, T-AOC assay kit and MDA assay kit were purchased from Shanghai yuanye Bio-Technology Co., Ltd (Shanghai, China).

\section{Real-time qPCR}

After blood samples collection, liver, spleen, ileum and cecum samples were harvested for interferon gamma (IFN- $\gamma$ ) and tumor necrosis factor-alpha (TNF-a) mRNA expression evaluation. Total RNA was extracted from these tissues using RNAiso Plus (Takara, Beijing, China) and reverse transcribed into cDNA with PrimeScript ${ }^{\text {TM }}$ RT reagent Kit with gDNA Eraser (Perfect Real Time) (Takara, Beijing, China) according to the 
manufacturer's protocol. The primers used in the study were synthesized by Sangon Biotech (Shanghai) Co., Ltd. (Shanghai, China) and primer sequences are summarized in Table 2. The Real-time qPCR reactions were performed using a SYBR Premix EX Taq Kit (Takara, Beijing, China) in a 7500 Fast Real-Time PCR System (Thermo Fisher). b-actin was used as a housekeeping gene. The relative mRNA expression levels of the target genes compared to the housekeeping gene were calculated using the $2^{-\Delta \Delta C t}$ method.

\section{Sample Collection and DNA Extraction}

On day 14 and day 28 , a total of 48 hens were randomly selected (twelve hens per group) and euthanized to collect ileal and cecal contents. The samples were named as 14-d ileum control group (14IA), 14-d ileum Astragalus group (14IB), 14-d ileum L. Plantarum group (14IC), 14-d ileum fermented Astragalus group (14ID), 14-d cecum control group (14CA), 14-d cecum Astragalus group (14CB), 14-d cecum L. Plantarum group (14CC), 14-d cecum fermented Astragalus group (14CD), 28-d ileum control group (28IA), 28-d ileum Astragalus group (28IB), 28-d ileum L. Plantarum group (28IC), 28-d ileum fermented Astragalus group (28ID), 28-d cecum control group (28CA), 28-d cecum Astragalus group (28CB), 28-d cecum L. Plantarum group (28CC), and 28-d cecum fermented Astragalus group (28CD). All collected samples were immediately stored at $-20^{\circ} \mathrm{C}$ until extraction. DNA extraction was performed with a commercial DNA extraction kit (Tiangen Biotech Corporation, Beijing, China) and quantified by a Qubit 2.0 fluorometer (Invitrogen Corporation, Carlsbad, CA, USA). The extracted DNA was qualitatively assessed by $0.8 \%$ agarose gel electrophoresis and spectrophotometry (optical density at $260 / 280 \mathrm{~nm}$ ) and stored at $-20^{\circ} \mathrm{C}$ until further analysis.

\section{S rRNA Gene Sequencing and Analysis}

For amplicon library generation, the V4 region of the 16S rRNA gene of all DNA samples was amplified with gene-specific primers (F: 5'- AYTGGGYDTAAAGNG-3'; R: 5'-TACNVGGGTATCTAATCC-3'). PCR amplifications were performed using Q5 high-fidelity PCR DNA polymerase (NEB) and completed under the following conditions: a pre-denaturation at $98^{\circ} \mathrm{C}$ for $30 \mathrm{~s} ; 27$ cycles of $98^{\circ} \mathrm{C}$ for $15 \mathrm{~s}, 50^{\circ} \mathrm{C}$ for $30 \mathrm{~s}$, and $72^{\circ} \mathrm{C}$ for $30 \mathrm{~s}$; a final extension at $72^{\circ} \mathrm{C}$ for $5 \mathrm{~min}$. Amplicons were purified using the Axygen AP-GX-250G AxyPrepTM DNA Gel Extraction Kit (Corning Life Sciences, Corning, NY, USA). DNA libraries were validated and quantified using the TruSeq Nano DNA LT Library Preparation Kit (Illumina, San Diego, CA, USA) and Quant-iT ${ }^{\mathrm{TM}}$ PicoGreen ${ }^{\text {TM }}$ dsDNA Assay Kit (Invitrogen Corporation, Carlsbad, CA, USA). After quantification, the barcoded V4 amplicons were pooled to a final concentration of $2 \mathrm{nmol} / \mathrm{L}$ and sequenced using an Illumina MiSeq platform to generate $300 \mathrm{bp}$ paired-end reads. Raw reads were quality-filtered to remove any reads less than 150 bp using Quantitative Insights into Microbial Ecology (QIIME) version 1.8 [16] and clustered into Operational Taxonomic Units (OTUs) based on a 97\% similarity threshold. The representative sequence was chosen based on the abundance and was aligned under a given taxonomic classification using the Greengenes database, and low abundance OTUs of archaea and eukaryotes were removed [17]. Alphadiversity was calculated with Chao1 and ACE estimators, Shannon and Simpson indices. Partial least squares discriminant analysis (PLS-DA) was performed using QIIME software package v1.8 to discriminate 
between different groups (day 14 and day 28) and to establish b-diversity. The sequences generated in this study have been deposited in the National Center for Biotechnology Information sequence read archive (https://www.ncbi.nlm.nih.gov/biosample) under the accession number SRA: PRJNA533918.

\section{Statistical Analysis}

Only for genes mRNA expression assay, data were analyzed and graphed using GraphPad Prism 6.00 (GraphPad Software), and significance levels are indicated as: * $P<0.05, * * P<0.01, * \star * P<0.001, * \star \star *$ $P<0.0001$. All other statistical analyses were performed by one-way analysis of variance using SPSS 24.0 software, and all data were expressed as means \pm SD, with $P<0.05$ considered statistically significant.

\section{Results}

\section{Hen Productivity and Egg Quality}

The effects of different dietary supplements on the laying hen production performance and egg quality are listed in Table 3 and 4. During day 1 to day 14, there were no differences in the laying rate and FCR among four groups ( $P>0.05$ ), with hens fed with fermented Astragalus had the highest laying rate. During day 15 to day 28, hens fed with fermented Astragalus had the highest laying rate, 7.14\% higher than that of the control group $(P<0.05)$. Although the differences were not statistically significant $(P>0.05)$, laying rate of the Astragalus group and L. Plantarum group were also increased by $3.25 \%$ and $2.99 \%$, respectively in comparison with the control group. Furthermore, the FCR of the fermented Astragalus group was reduced by $6.6 \%$ compared with that of the control group $(P<0.05)$, while the FCR of the Astragalus group and $L$. Plantarum group displayed no significant differences as compared with the controls $(P>0.05)$. In addition, no significant differences in the phenotype of the egg quality including ESI, ESS, EST, AH, YC, HU and YW were observed among dietary treatments, suggesting that dietary supplements have no significant effects on egg quality in this study. Therefore, we identified that dietary supplementation of fermented Astragalus can markedly improve egg production and decrease FCR, and the effect is substantially superior to that of Astragalus and L. Plantarum.

\section{Serum Antioxidant Indices}

The effects of different dietary supplements on the laying hen antioxidant status are listed in Table 5. The data indicated that all dietary supplementation did not have an effect on the biomarkers of antioxidative stress at day 14 ( $P>0.05)$. However, serum CAT, GSH-Px, SOD and T-AOC concentrations were increased by $61.5 \%, 62.4 \%, 68.0 \%$ and $52.6 \%(P<0.05)$ at the end of experimentation in the fermented Astragalus group as compared with the controls. No statistically significant differences were observed for CAT, GSH-Px and SOD amoug the control, Astragalus and L. plantarum groups ( $P>0.05)$. Among the effects of different dietary supplements on MDA activity in serum of laying hens, hens fed with fermented Astragalus, Astragalus diet were significantly decreased by $54.7 \%$ and $43.0 \%$ than that of the control treatment $(P<0.05)$; treatment with $L$. plantarum diet did not dramatically differ from the control treatment $(P>0.05)$. The results 
presented above show that dietary supplementation of fermented Astragalus can markedly improve laying hen antioxidant status, and the effect is significantly superior to that of Astragalus and L. Plantarum.

\section{IFN-Y and TNF-a mRNA expression}

The expression levels of IFN- $y$ and TNF-a mRNA in the liver, spleen, ileum and cecum were assessed at 14 and 28 day. As shown in Figure 1 and 2, the addition of fermented Astragalus to the diets respectively increased the mRNA content on day 14 of IFN- $y$ and TNF- $\alpha$ in the ileum by 1.7 -fold $(P<0.01)$ and 3.1-fold $(P<0.001)$, and the mRNA content on day 14 of IFN-y in the cecum by 2.1 -fold $(P<0.01)$. Interestingly, we found that the highest amount of IFN- $\gamma$ and TNF-a mRNA in the liver, spleen, ileum and cecum were present in the fermented Astragalus group at 28 day.

\section{Sequencing Output}

A total of 48 intestinal content samples were analysed by $16 \mathrm{~S}$ rRNA gene sequencing and produced a total of 2,006,223 high-quality sequences with an average of 41,796 reads. After OUT clustering at $97 \%$ sequence identity, a total of 216,116 OTUs were classified into 49,235 phyla, 48,677 classes, 48,634 orders, 40,101 families, 24,072 genera and 4,995 species (Figure 3).

\section{Diversity of Intestinal Microflora}

The a-diversity of ileal and cecal microbiota of four groups at different days are shown in Table 6 . For bacteria on day 14, fermented Astragalus treatment reduced the Chao1 and ACE index in the cecum in comparison to the control treatment suggesting that fermented Astragalus decreased the richness of the bacterial communities. On day 28, the fermented Astragalus treatment increased the diversity estimators (Shannon and Simpson) of the bacterial community in the ileum. PLS-DA was performed to evaluate the similarity (b-diversity) of microbial community structure among groups (Figure 4). PLS-DA plot defined groups where the samples from different groups occupied distinct positions.

\section{Composition of Intestinal Microflora}

A total of 20 phyla were identified within the intestinal microbiota among 48 samples as shown in Figure 5 . There were 3 major groups of the intestinal microbiota, including Firmicutes, Bacteroidetes and Proteobacteria. The relative abundance (\%) of cecal bacterial phyla of hens fed with different dietary supplements was presented in approximately the same amount on days 14 and 28 . On day 28 , fermented Astragalus led to a reduced abundance of ileal Firmicutes, with an increased abundance of ileal Bacteroidetes and Proteobacteri. Genus level analysis showed that the Lactobacillus and Bacteroides accounted for the largest proportion of the intestinal microbiota as shown in Figure 6. Lactobacillus showed high abundance in the ileum, and extremely low abundance in the cecum. In contrast, Bacteroides showed high abundance in the cecum, and extremely low abundance in the ileum. On day 14, fermented Astragalus 
addition increased the abundance of cecal Bacteroides by $5.06 \%$ as compared with the control, with no significant influence on the abundance of ileal Lactobacillus. On day 28, fermented Astragalus addition significantly decreased the abundance of ileal Lactobacillus by $48.51 \%$ as compared with the control, with no significant influence on the abundance of cecal Bacteroides.

\section{Discussion}

The present study was undertaken to investigate the effects of L. Plantarum fermented Astragalus supplementation on the performance, egg quality, antioxidant status of serum and gut microbiota in laying hens. When taking out the feeding trial, we observed that diets supplemented with fermented Astragalus increased egg production rate $(P<0.05)$ and decreased feed conversion rate $(P<0.05)$, which may likely be attributed to the improvement of laying hen health status. In vivo, free radicals are harmful by-products generated during normal cellular metabolism, and are prone to attack unsaturated fatty acid on the biological membrane, triggering lipid oxidation and lipid peroxides accumulation that result in impairment of organism health [18]. The antioxidant enzymes CAT, GSH-Px and SOD are associated with free radical scavenging to protect cells from oxidative damage [19]. In the present study, supplementation with fermented Astragalus resulted in a significantly highest levels of CAT, GSH-Px, SOD and T-AOC and lowest level of MDA in the serum $(P<0.05)$ on day 28. Our findings are consistent with our previous studies on broilers [20], indicating that Astragalus fermented by L. plantarum can enhance the antioxidant ability of both broilers and laying hens.

Nowadays, Astragalus polysaccharide has attracted rising interests for its anti-cancer effects. Previous study has observed that Astragalus polysaccharide can significantly enhance the proliferation of spleen lymphocytes and increase phagocytosis of peritoneal macrophages in mice and is capable of up-regulating the expression of IL-2, TNF- $\alpha$ and IFN- $\gamma$ in peripheral blood [21]. IFN- $\gamma$ and TNF- $\alpha$ are cytokines possessing antitumor and immunomodulatory properties and are essential for host immune responses against infection or tissue injury [22]. At the end of our feeding trial (on day 28), L. plantarum merely increased the mRNA expression of ileal TNF-a, Astragalus increased the mRNA expression of splenic and cecal IFN- $\gamma$ and that of hepatic, splenic and cecal TNF-a. Interestingly, fermented Astragalus significantly increased the mRNA expression of both IFN- $y$ and TNF- $a$ in all the liver, spleen, ileum, and cecum. However, there are comparatively few findings to date regarding the impact of $L$. Plantarum fermented Astragalus on host immune responses. We speculate that components and metabolites of Astragalus are changed after fermentation and more effective components can enhance the body's immune function by increasing the expression of cytokines. Certainly, further investigations will be required to fully illustrate the intrinsic molecular mechanism.

Intestinal microbiota plays an major role in maintaining host health, immunity and production performance, it has become a research hotspot in recent years [23]. In this study, we also evaluated the effect of fermented Astragalus on intestinal microbiota of laying hens. Our results showed that fermented Astragalus addition increases the diversity of ileal bacterial community with the increase of feeding time. Furthermore, at the phylum level, Firmicutes, Bacteroidetes and Proteobacteria were the most dominant phyla in the intestinal microbiota of hens, which is consistent with previous studies [23, 24]. Interestingly, fermented 
Astragalus addition led to a reduced abundance of ileal Firmicutes, with an increased abundance of ileal Bacteroidetes and Proteobacteria. We speculate that increased diversity of ileal bacterial community might be explained by the fact that the abundance of ileal Firmicutes was reduced to enhance the abundance of other phyla. At the genus level, Lactobacillus as the largest proportion of ileal microbiota of hens is generally highly relevant to feed digestibility [25]. However, fermented Astragalus addition significantly decreased the abundance of ileal Lactobacillus by $48.51 \%$ as compared with the control at 28 days. These results were totally different from our previous report on the effect of fermented Astragauls on the broiler chicken fecal microbiota, which found that the count of Lactobacillus was increased in chickens fed fermented Astragalus as compared with those in the control group. Those factors responsible for the differences should be further studied.

\section{Conclusions}

This study suggested that L. Plantarum fermented Astragalus as an efficient dietary additive could significantly promote the production performance, antioxidant capacity and ileal microbiota diversity of laying hens during the late laying period. A higher expression level of IFN- $y$ and TNF- $\alpha$ in the liver, spleen, ileum and cecum of laying hens supplemented with fermented Astragalus indicates a particular role of fermented Astragalus on the innate immune system, and this needs a comprehensive investigation in the future to fully illustrate the exact mechanism.

\section{Abbreviations}

L. Plantarum: Lactobacillus Plantarum; CAT: catalase; GSH-Px: glutathione peroxidase; SOD: superoxide dismutase; T-AOC: total antioxidant capacity; MDA: malondialdehyde; CFU: colony forming unit; FCR: feed conversion ratio; ESI: egg shape index; ESS: eggshell strength; EST: eggshell thickness; AH: albumen height; HU: haugh unit; YC: yolk color; YW: yolk weight; IFN- $Y$ : interferon gamma; TNF-a: tumor necrosis factoralpha; OTUs: Operational Taxonomic Units; PLS-DA: Partial least squares discriminant analysis.

\section{Declarations}

Ethics approval and consent to participate

The animal experiments were conducted in accordance with the Guidelines for the Care and Use of Experimental Animals established and approved by the Laboratory Animal Management Committee of Henan University of Animal Husbandry and Economy.

Consent for publication

Not applicable

Availability of data and materials 
The datasets generated during this study have been deposited in the National Center for Biotechnology Information sequence read archive (https://www.ncbi.nlm.nih.gov/biosample) under the accession number SRA: PRJNA533918.

Competing interests

The authors declare that they have no competing interests.

Funding

This study was supported by the Key Discipline of Preventive Veterinary Medicine of Henan University of Animal Husbandry and Economy (mxk2016102), the Research and Innovation Team of Henan University of Animal Husbandry and Economy (2018KYTD13) and the Henan Science and Technology Research Project (192102110076).

Authors' Contributions

HXQ and HTS contributed to conception and design of the study. HTS, BYW and CZB performed the experiments. HXQ and BYW performed the statistical analysis. YQH and HXQ wrote the manuscript. All authors contributed to manuscript revision, read, and approved the submitted version

Acknowledgements

Not applicable

\section{Reference}

1. Vandemaele F, Vereecken M, Derijcke J, Goddeeris BM: Incidence and antibiotic resistance of pathogenic Escherichia coli among poultry in Belgium. Vet Rec 2002, 151(12):355-356.

2. BP W, SL R, BB F: Shifting the balance: antibiotic effects on host-microbiota mutualism. Nature reviews Microbiology 2011, 9(4):233-243.

3. Xu J, Chen HB, Li SL: Understanding the Molecular Mechanisms of the Interplay Between Herbal Medicines and Gut Microbiota. Medicinal Research Reviews 2017, 37(5).

4. Wang CZ, Li WJ, Tao R, Ye JZ, Zhang HY: Antiviral activity of a nanoemulsion of polyprenols from ginkgo leaves against influenza A H3N2 and hepatitis B virus in vitro. Molecules 2015, 20(3):51375151.

5. Patra JK, Das G, Baek KH: Chemical Composition and Antioxidant and Antibacterial Activities of an Essential Oil Extracted from an Edible Seaweed, Laminaria japonica L. Molecules 2015, 20(7):1209312113.

6. Zhu H, Cao G, Cai H, Cai B, Hu J: Rapid and undamaged analysis of crude and processed Radix Scrophulariae by Fourier transform infrared spectroscopy coupled with soft independent modeling of class analogy. Pharmacogn Mag 2014, 10(39):265-270. 
7. Ai S, Tang W, Guo RL, Li JQ, Yang W, He ZG: [Research progress on Chinese herbal medicine fermentation and profile of active substances derived]. Zhongguo Zhong Yao Za Zhi 2019, 44(6):11101118.

8. Li X, Qu L, Dong Y, Han L, Liu E, Fang S, Zhang Y, Wang T: A review of recent research progress on the astragalus genus. Molecules 2014, 19(11):18850-18880.

9. Kim JH, Kim MH, Yang G, Huh Y, Kim SH, Yang WM: Effects of topical application of Astragalus membranaceus on allergic dermatitis. Immunopharmacol Immunotoxicol 2013, 35(1):151-156.

10. Kallon S, Li X, Ji J, Chen C, Xi Q, Chang S, Xue C, Ma J, Xie Q, Zhang Y: Astragalus polysaccharide enhances immunity and inhibits H9N2 avian influenza virus in vitro and in vivo. J Anim Sci Biotechnol 2013, 4(1):22.

11. Shahzad M, Shabbir A, Wojcikowski K, Wohlmuth H, Gobe GC: The Antioxidant Effects of Radix Astragali (Astragalus membranaceus and Related Species) in Protecting Tissues from Injury and Disease. Curr Drug Targets 2016, 17(12):1331-1340.

12. Qin Q, Niu J, Wang Z, Xu W, Qiao Z, Gu Y: Astragalus membranaceus inhibits inflammation via phospho-P38 mitogen-activated protein kinase (MAPK) and nuclear factor (NF)-KB pathways in advanced glycation end product-stimulated macrophages. Int J Mol Sci 2012, 13(7):8379-8387.

13. Sheih IC, Fang TJ, Wu TK, Chang CH, Chen RY: Purification and properties of a novel phenolic antioxidant from Radix astragali fermented by Aspergillus oryzae M29. J Agric Food Chem 2011, 59(12):6520-6525.

14. Qiao H, Zhang X, Shi H, Song Y, Bian C, Guo A: Assessment of the physicochemical properties and bacterial composition of Lactobacillus plantarum and Enterococcus faecium-fermented Astragalus membranaceus using single molecule, real-time sequencing technology. Sci Rep 2018, 8(1):11862.

15. Qiao H, Zhang L, Shi H, Song Y, Bian C: Astragalus affects fecal microbial composition of young hens as determined by $16 \mathrm{~S}$ rRNA sequencing. $A M B$ Express 2018, 8(1):70.

16. Caporaso JG, Kuczynski J, Stombaugh J, Bittinger K, Bushman FD, Costello EK, Fierer N, Peña AG, Goodrich JK, Gordon JI et al: QIIME allows analysis of high-throughput community sequencing data. Nat Methods 2010, 7(5):335-336.

17. Bokulich NA, Subramanian S, Faith JJ, Gevers D, Gordon JI, Knight R, Mills DA, Caporaso JG: Qualityfiltering vastly improves diversity estimates from Illumina amplicon sequencing. Nat Methods 2013, 10(1):57-59.

18. Fang YZ, Yang S, Wu G: Free radicals, antioxidants, and nutrition. Nutrition 2002, 18(10):872-879.

19. Zhang Z, Liu D, Yi B, Liao Z, Tang L, Yin D, He M: Taurine supplementation reduces oxidative stress and protects the liver in an iron-overload murine model. Mol Med Rep 2014, 10(5):2255-2262.

20. Qiao H, Song Y, Shi H, Bian C: Fermented Astragalus in diet altered the composition of fecal microbiota in broiler chickens. AMB Express 2018, 8(1):151.

21. Li W, Hu X, Wang S, Jiao Z, Sun T, Liu T, Song K: Characterization and anti-tumor bioactivity of astragalus polysaccharides by immunomodulation. Int J Biol Macromol 2020, 145:985-997. 
22. Li Q, Liu C, Yue R, El-Ashram S, Wang J, He X, Zhao D, Zhou X, Xu L: cGAS/STING/TBK1/IRF3 Signaling Pathway Activates BMDCs Maturation Following Mycobacterium bovis Infection. Int J Mol Sci 2019, 20(4).

23. Yeoman CJ, Chia N, Jeraldo P, Sipos M, Goldenfeld ND, White BA: The microbiome of the chicken gastrointestinal tract. Anim Health Res Rev 2012, 13(1):89-99.

24. Danzeisen JL, Kim HB, Isaacson RE, Tu ZJ, Johnson TJ: Modulations of the chicken cecal microbiome and metagenome in response to anticoccidial and growth promoter treatment. PLOS One 2011, 6(11):e27949.

25. Yan W, Sun C, Yuan J, Yang N: Gut metagenomic analysis reveals prominent roles of Lactobacillus and cecal microbiota in chicken feed efficiency. Sci Rep 2017, 7:45308.

\section{Tables}

Table 1 Composition and nutrient levels of the basal diet (air-dry basis \%)

\begin{tabular}{|c|c|c|c|}
\hline Ingredients & Content & Nutrient levels & Content \\
\hline Corn & 61.4 & $\mathrm{ME} /(\mathrm{MJ} / \mathrm{kg})^{2}$ & 11.01 \\
\hline $\begin{array}{l}\text { Soybean meal } \\
\text { Wheat bran } \\
\text { Soybean oil } \text { CaHPO }_{4}\end{array}$ & $\begin{array}{l}23.8 \\
2.0 \\
0.6 \\
1.3\end{array}$ & $\begin{array}{l}\mathrm{CP} \\
\mathrm{Ca} \\
\mathrm{TP} \\
\mathrm{AP}\end{array}$ & $\begin{array}{l}15.49 \\
3.50 \\
0.56 \\
0.35\end{array}$ \\
\hline $\begin{array}{l}\text { Limestone } \\
\text { NaCl } \\
\text { Premix }{ }^{1}\end{array}$ & $\begin{array}{l}8.6 \\
0.3 \\
2.0\end{array}$ & $\begin{array}{l}\text { Lys } \\
\text { Met+Cys }\end{array}$ & $\begin{array}{l}0.74 \\
0.51\end{array}$ \\
\hline Total & 100.0 & & \\
\hline
\end{tabular}

${ }^{1}$ The premix provided the following per kilogram of the diettVA $11,000 \mathrm{IU}, \mathrm{VD}_{3} 3,200 \mathrm{IU}, \mathrm{VE} 25 \mathrm{IU}, \mathrm{VK}_{3} 2.2$ $\mathrm{mg}, \mathrm{VB}_{1} 1.5 \mathrm{mg}, \mathrm{VB}_{2} 3.5 \mathrm{mg}, \mathrm{VB}_{12} 3 \mathrm{mg}$, nicotinic $28 \mathrm{mg}$, calcium pantothenate $8.5 \mathrm{mg}$, biotin $0.5 \mathrm{mg}$, choline 255 mg, Fe 55 mg, Zn 62 mg, Cu 6 mg, Se 0.20 mg.

${ }^{2}$ ME was a calculated value, while the others were measured values.

Table 2 Primers used for quantitative real-time PCR analysis

\begin{tabular}{lll}
\hline Primers & Sequences 5' 3' & Size \\
\hline IFN-y-F & AACAACCTTCCTGATGGCGT & $107 \mathrm{bp}$ \\
IFN- $-\mathrm{r}-\mathrm{R}$ & TGAAGAGTTCATTCGCGGCT & \\
b-actin-F & TATGTGCAAGGCCGGTTCG & $170 \mathrm{bp}$ \\
b-actin-R & CAATGGGGTACTTCAGGGTCAG & \\
TNF-a-F & GCCCTTCCTGTAACCAGATG & $71 \mathrm{bp}$ \\
TNF-a-R & ACACGACAGCCAAGTCAACG & \\
\hline
\end{tabular}

Table 3 Effects of different dietary supplements on the production performance of laying hens 


\begin{tabular}{|c|c|c|c|c|c|c|c|}
\hline Parameters & & Control & Astragulas & L. plantarum & $\begin{array}{l}\text { Fermented } \\
\text { Astragulas }\end{array}$ & $P$-value & SEM \\
\hline \multirow{2}{*}{$\begin{array}{l}\text { Laying rate, } \\
\%\end{array}$} & $1-14 d$ & $87.50 \pm 3.45$ & $89.17 \pm 2.08$ & $87.50 \pm 2.70$ & $90.60 \pm 3.80$ & 0.349 & 1.376 \\
\hline & 15- & $88.96 \pm 3.76 a$ & $92.21 \pm 2.75 a b$ & $91.95 \pm 3.83 a b$ & $96.10 \pm 1.03 b$ & 0.017 & 1.368 \\
\hline \multirow[t]{2}{*}{ FCR } & $1-14 d$ & $2.02 \pm 0.08$ & $1.94 \pm 0.05$ & $2.02 \pm 0.10$ & $1.95 \pm 0.08$ & 0.224 & 0.037 \\
\hline & $\begin{array}{l}15- \\
28 d\end{array}$ & $1.81 \pm 0.08 b$ & $1.75 \pm 0.06 \mathrm{ab}$ & $1.77 \pm 0.11 \mathrm{ab}$ & $1.69 \pm 0.05 a$ & 0.172 & 0.035 \\
\hline
\end{tabular}

Note: FCR, feed conversion ratio. Different lowercase letters in the same row indicate significant difference $(P<0.05)$, and the same letters or no letters indicate no significant difference $(P>0.05)$ (same as below).

Table 4 Effects of different dietary supplements on the egg quality

\begin{tabular}{lllllll}
\hline Parameters & Control & Astragulas & L. plantarum & Fermented Astragulas & $P$-value & SEM \\
ESI & $1.29 \pm 0.02$ & $1.29 \pm 0.03$ & $1.29 \pm 0.03$ & $1.29 \pm 0.03$ & 0.961 & 0.012 \\
ESS $(\mathrm{kg} / \mathrm{N})$ & $3.59 \pm 0.41$ & $4.04 \pm 0.33$ & $3.87 \pm 0.32$ & $3.90 \pm 0.40$ & 0.302 & 0.164 \\
EST $(\mathrm{mm})$ & $0.344 \pm 0.013$ & $0.344 \pm 0.011$ & $0.346 \pm 0.008$ & $0.348 \pm 0.008$ & 0.927 & 0.005 \\
AH $(\mathrm{mm})$ & $8.74 \pm 0.43 \mathrm{ab}$ & $8.74 \pm 0.63 \mathrm{ab}$ & $8.47 \pm 0.51 \mathrm{a}$ & $9.21 \pm 0.33 \mathrm{~b}$ & 0.162 & 0.219 \\
YC & $5.15 \pm 0.32$ & $5.00 \pm 0.28$ & $5.44 \pm 0.21$ & $5.32 \pm 0.40$ & 0.160 & 0.139 \\
HU & $92.84 \pm 1.90$ & $92.16 \pm 3.42$ & $92.10 \pm 2.82$ & $94.75 \pm 1.61$ & 0.183 & 1.138 \\
YW & $15.75 \pm 0.37$ & $15.47 \pm 0.32$ & $15.60 \pm 0.22$ & $15.27 \pm 0.73$ & 0.417 & 0.202 \\
\hline $28 \mathrm{~d}$ & $1.28 \pm 0.02$ & $1.28 \pm 0.01$ & $1.29 \pm 0.01$ & $1.29 \pm 0.03$ & 0.452 & 0.008 \\
ESI & & & & & 0.625 & 0.185 \\
ESS $(\mathrm{kg} / \mathrm{N})$ & $4.31 \pm 0.37$ & $4.34 \pm 0.60$ & $4.20 \pm 0.16$ & $4.54 \pm 0.41$ & 0.396 & 0.007 \\
EST $(\mathrm{mm})$ & $0.375 \pm 0.014$ & $0.392 \pm 0.020$ & $0.389 \pm 0.013$ & $0.387 \pm 0.018$ & 0.676 & 0.173 \\
AH (mm) & $8.80 \pm 0.40$ & $8.92 \pm 0.43$ & $8.85 \pm 0.24$ & $9.08 \pm 0.44$ & 0.435 & 0.170 \\
YC & $5.59 \pm 0.39$ & $5.55 \pm 0.47$ & $5.22 \pm 0.12$ & $5.44 \pm 0.45$ & 0.763 & 0.895 \\
HU & $92.57 \pm 2.19$ & $92.80 \pm 2.42$ & $92.74 \pm 1.03$ & $93.80 \pm 2.07$ & 0.556 & 0.222 \\
YW & $15.40 \pm 0.39$ & $15.50 \pm 0.59$ & $15.78 \pm 0.38$ & $15.76 \pm 0.58$ & & \\
\hline
\end{tabular}

Note: ESI, egg shape index; ESS, eggshell strength; EST, eggshell thickness; $A H$, albumen height; HU, haugh unit; YC, yolk color; YW, yolk weight. 
Table 5 Effects of different dietary supplements on the antioxidant status of laying hens

\begin{tabular}{|c|c|c|c|c|c|c|}
\hline Parameters & Control & Astragulas & L. plantarum & $\begin{array}{l}\text { Fermented } \\
\text { Astragulas } \\
\end{array}$ & $P$-value & SEM \\
\hline \multicolumn{7}{|l|}{$14 \mathrm{~d}$} \\
\hline \multirow{6}{*}{$\begin{array}{l}\text { CAT } \\
\text { (U/mL) } \\
\text { GSH-Px } \\
\text { (U/L) } \\
\text { SOD } \\
(\mathrm{U} / \mathrm{mL}) \\
\text { T-AOC } \\
(\mathrm{U} / \mathrm{mL}) \\
\mathrm{MDA} \\
(\mathrm{nmol} / \mathrm{mL}) \\
28 \mathrm{~d}\end{array}$} & $43.90 \pm 2.46$ & $57.21 \pm 13.66$ & $76.80 \pm 44.15$ & $61.52 \pm 31.03$ & 0.445 & 13.960 \\
\hline & $90.80 \pm 14.36$ & $115.32 \pm 32.20$ & $145.76 \pm 79.19$ & $120.37 \pm 54.47$ & 0.450 & 23.989 \\
\hline & $276.62 \pm 44.10$ & $313.42 \pm 87.16$ & $321.99 \pm 91.99$ & $339.02 \pm 127.71$ & 0.757 & 43.954 \\
\hline & $12.25 \pm 2.13$ & $14.33 \pm 4.66$ & $15.40 \pm 4.63$ & $14.79 \pm 6.31$ & 0.753 & 2.211 \\
\hline & $6.93 \pm 0.52$ & $8.58 \pm 2.50$ & $8.71 \pm 2.62$ & $8.63 \pm 3.52$ & 0.734 & 1.276 \\
\hline & & & & & & \\
\hline \multirow{5}{*}{$\begin{array}{l}\text { CAT } \\
(\mathrm{U} / \mathrm{mL}) \\
\text { GSH-Px } \\
\text { (U/L) } \\
\text { SOD } \\
(\mathrm{U} / \mathrm{mL}) \\
\text { T-AOC } \\
(\mathrm{U} / \mathrm{mL}) \\
\mathrm{MDA} \\
(\mathrm{nmol} / \mathrm{mL})\end{array}$} & $47.93 \pm 2.41 a$ & $45.58 \pm 8.17 a$ & $86.63 \pm 17.73 a b$ & $124.58 \pm 66.85 b$ & 0.020 & 17.563 \\
\hline & $90.07 \pm 20.18 a$ & $108.12 \pm 31.99 a$ & $159.86 \pm 36.77 a b$ & $239.78 \pm 142.58 b$ & 0.043 & 35.992 \\
\hline & $230.98 \pm 29.88 a$ & $321.95 \pm 125.37 a$ & $473.54 \pm 125.13 a b$ & $722.33 \pm 418.70 b$ & 0.030 & 108.041 \\
\hline & $10.78 \pm 1.62 a$ & $12.81 \pm 2.82 a$ & $20.29 \pm 4.68 b$ & $22.72 \pm 7.16 \mathrm{~b}$ & 0.006 & 2.226 \\
\hline & $14.79 \pm 4.97 \mathrm{c}$ & $8.43 \pm 3.28 a b$ & $12.47 \pm 2.69 \mathrm{bc}$ & $6.70 \pm 1.04 a$ & 0.011 & 1.542 \\
\hline
\end{tabular}

Figures 
(A)

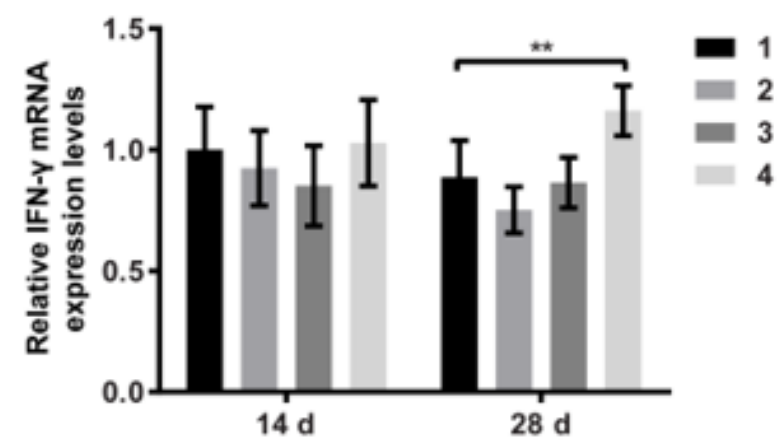

(C)

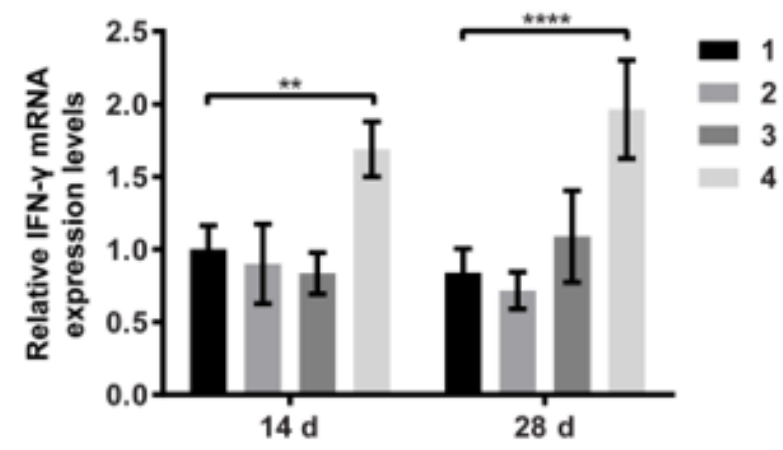

(B)

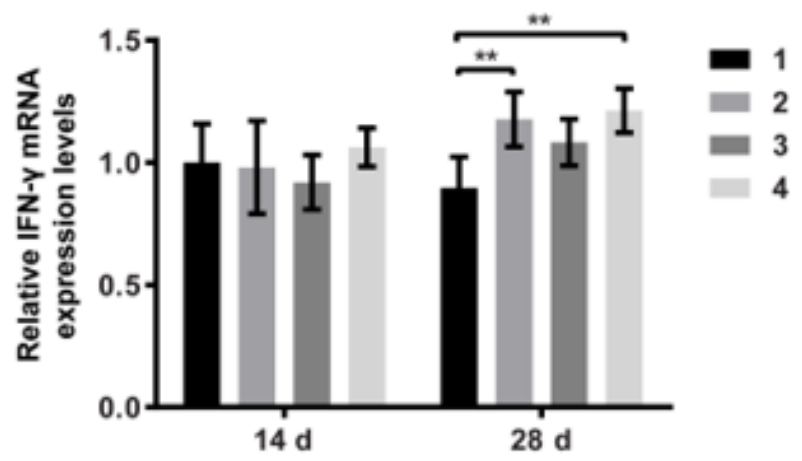

(D)

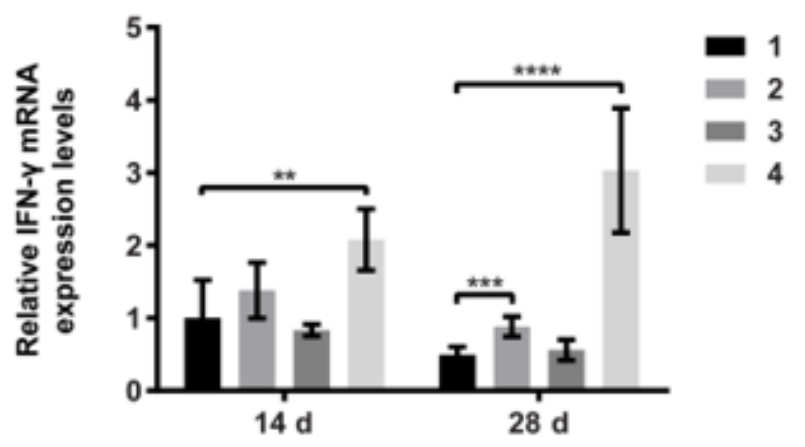

Figure 1

Effects of different dietary supplements on the relative mRNA expression of IFN- $\square$ of laying hens. (A) Expression levels of IFN-y mRNA in the liver at different time points; (B) Expression levels of IFN-y mRNA in the spleen at different time points; (C) Expression level of IFN-y mRNA in the ileum at different time points; (D) Expression level of IFN-y mRNA in the cecum at different time points; 1: the control group; 2: The Astragalus group; 3: The L. Plantarum group; 4: The fermented Astragalus group. 
(A)

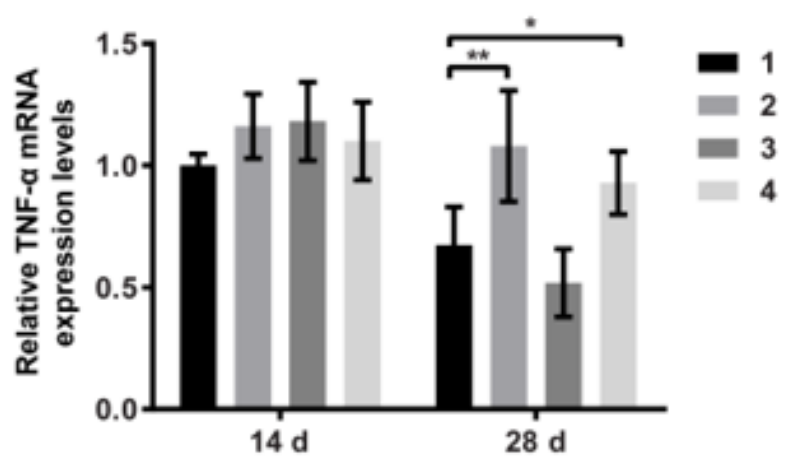

(C)

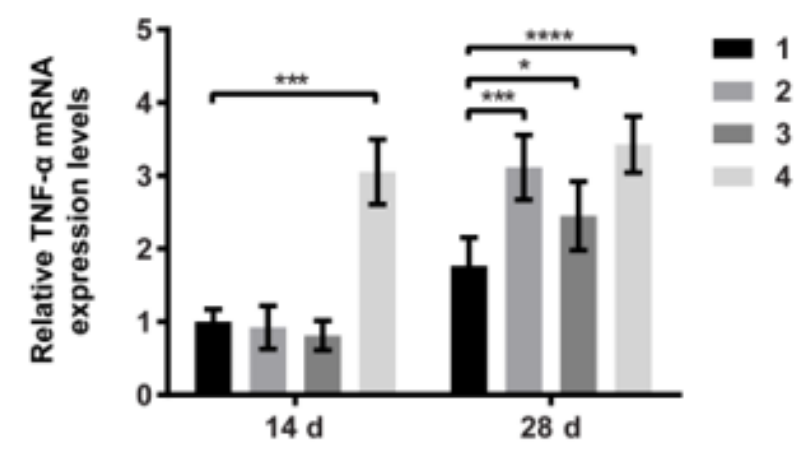

(B)

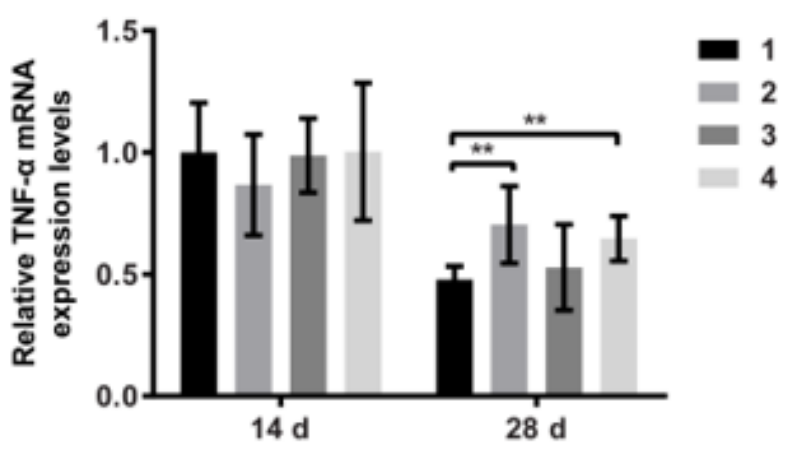

(D)

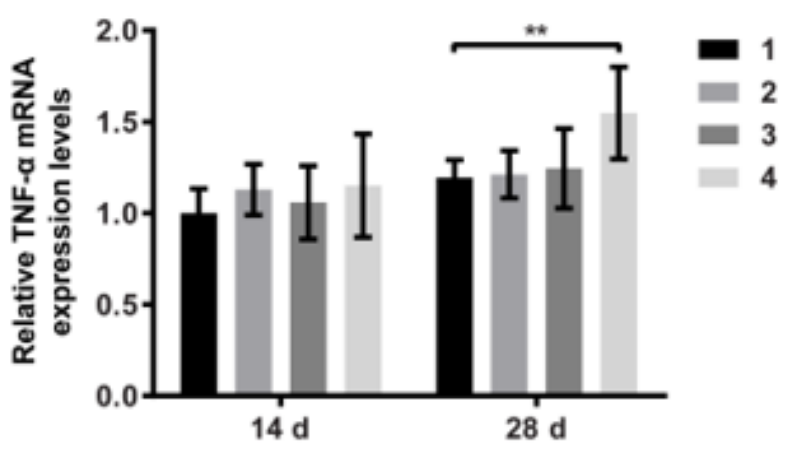

Figure 2

Effects of different dietary supplements on the relative mRNA expression of TNF-a of laying hens. (A) Expression levels of TNF-a mRNA in the liver at different time points; (B) Expression levels of TNF-a mRNA in the spleen at different time points; (C) Expression level of TNF-a mRNA in the ileum at different time points; (D) Expression level of TNF-a mRNA in the cecum at different time points; 1: the control group; 2: The Astragalus group; 3: The L. Plantarum group; 4: The fermented Astragalus group.

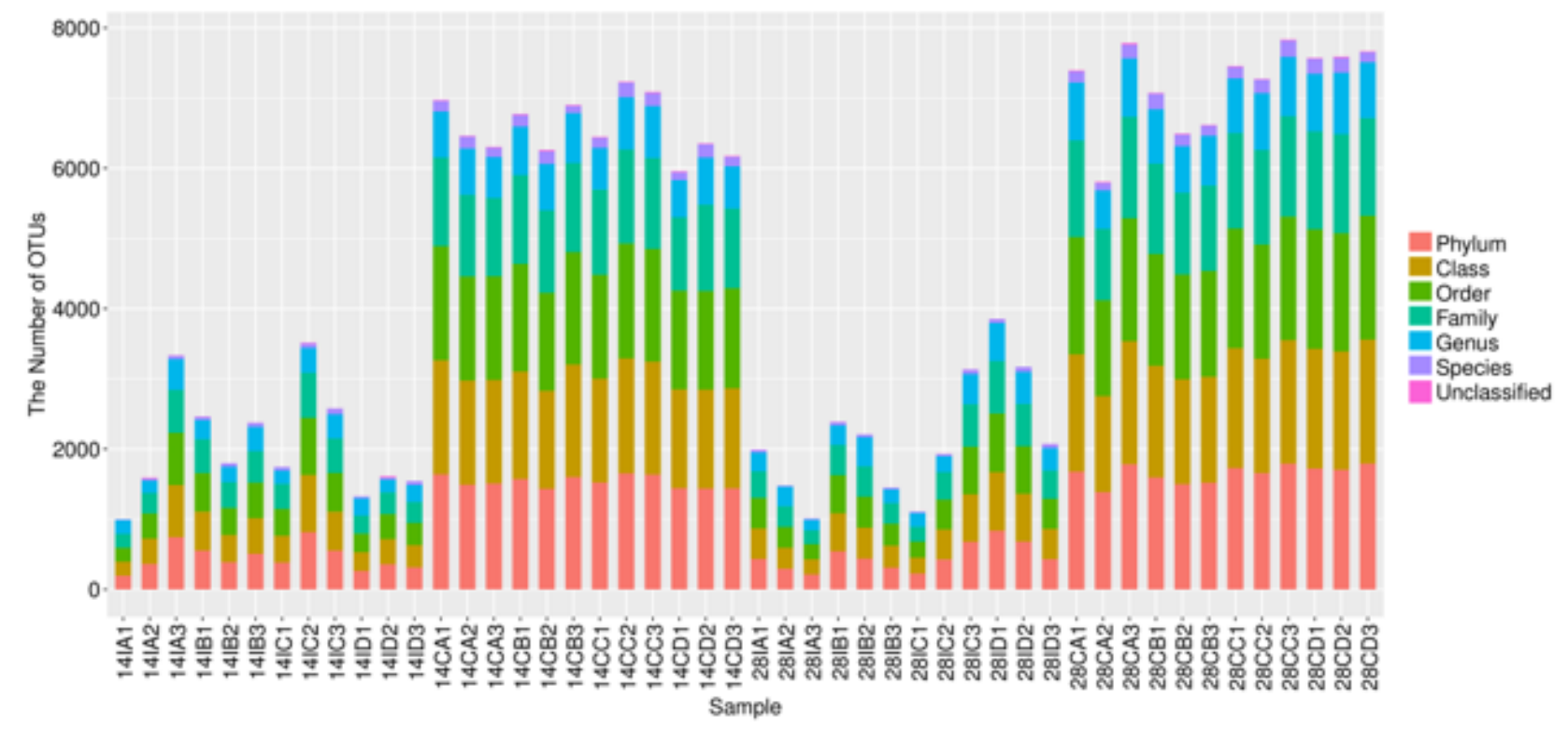


Figure 3

Number of identified taxa (from phyla to species) among the diverse groups.

\section{PLS-DA}

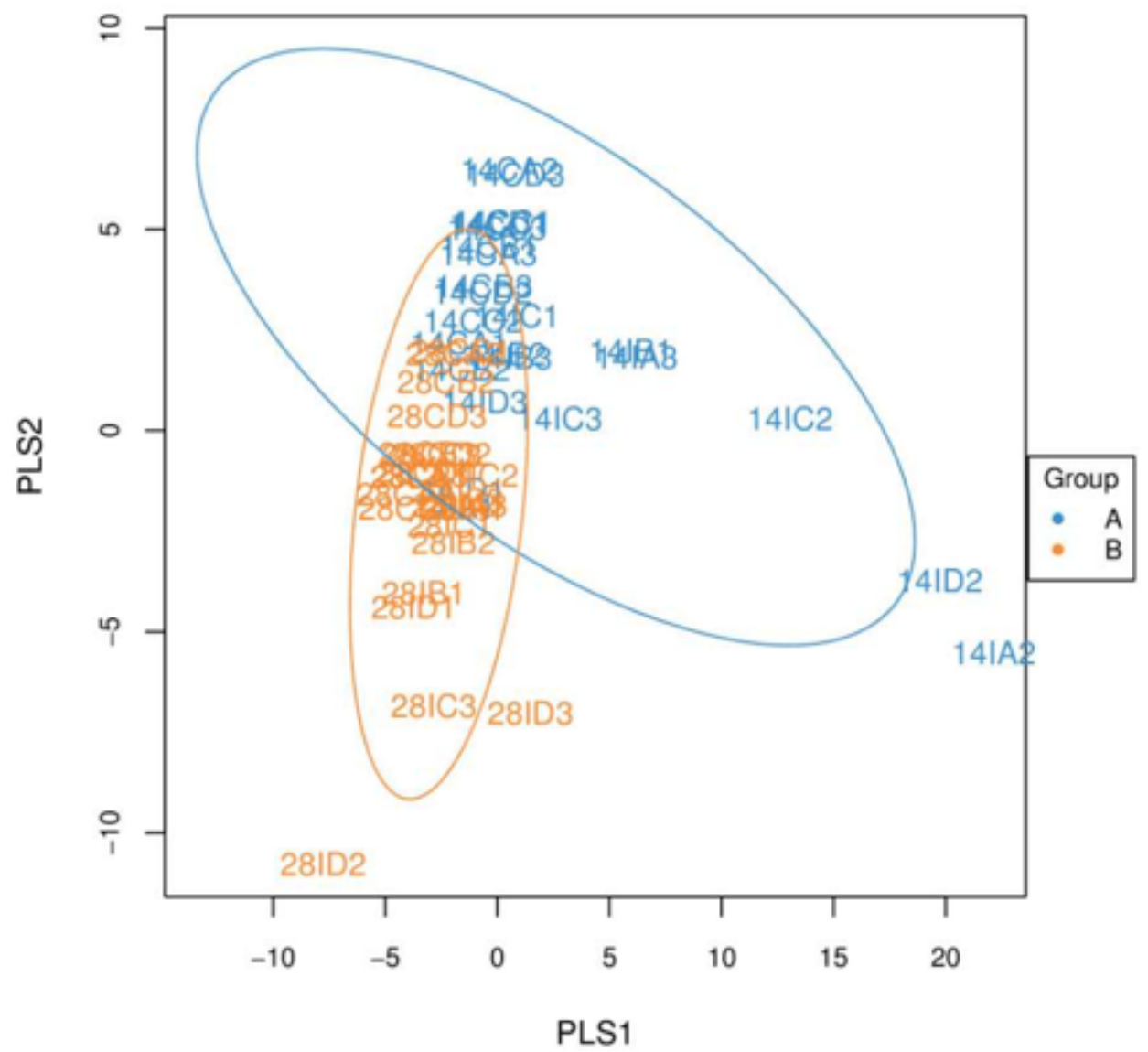

Figure 4

Partial least squares discriminant analysis (PLS-DA) of ileal and cecal microbiota among groups. A: 14-day sample groups; B: 28-day sample groups. 


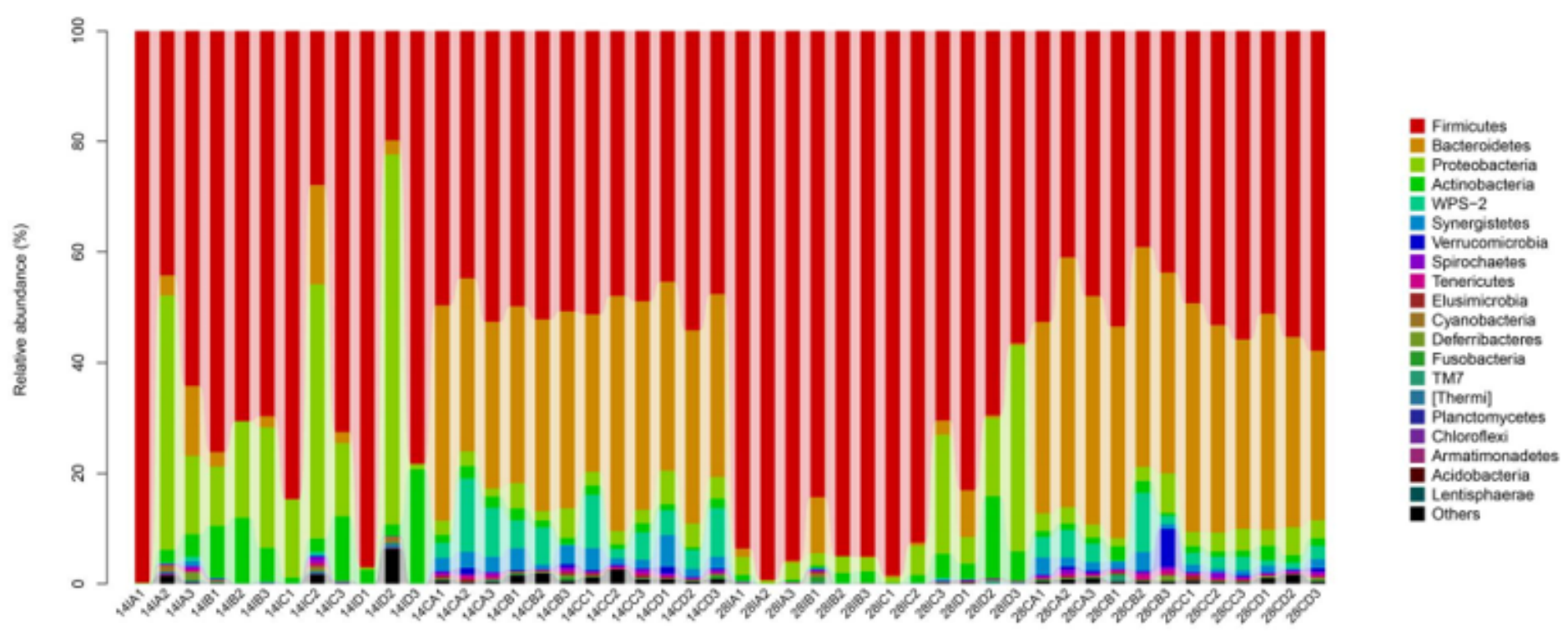

Figure 5

The phylum level distribution of ileal and cecal microbiota among groups. 14IA: 14-d ileum control group; 14IB: 14-d ileum Astragalus group; 14IC: 14-d ileum L. Plantarum group; 14ID: 14-d ileum fermented Astragalus group; 14CA: 14-d cecum control group; 14CB: 14-d cecum Astragalus group; 14CC: 14-d cecum L. Plantarum group; 14CD: 14-d cecum fermented Astragalus group; 28IA: 28-d ileum control group; 28IB: 28-d ileum Astragalus group; 28IC: 28-d ileum L. Plantarum group; 28ID: 28-d ileum fermented Astragalus group; 28CA: 28-d cecum control group; 28CB: 28-d cecum Astragalus group; 28CC: 28-d cecum L. Plantarum group; 28CD: 28-d cecum fermented Astragalus group.

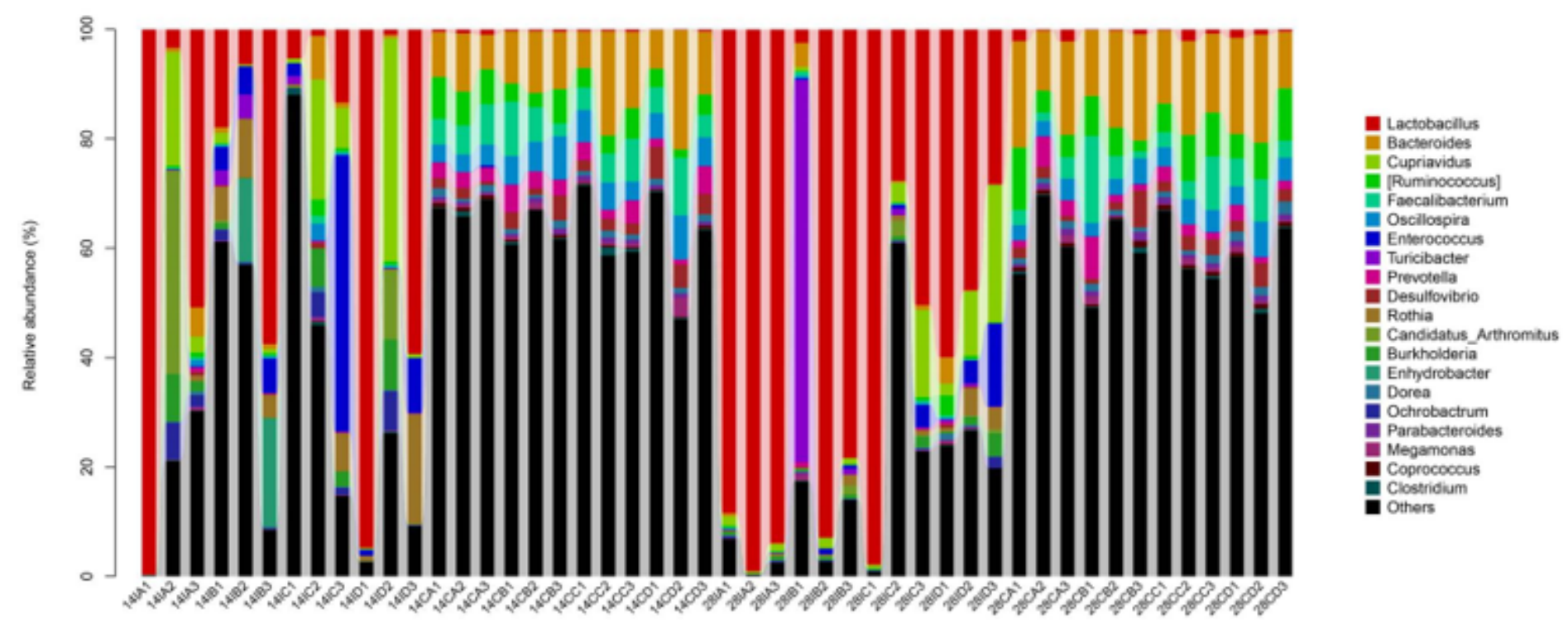

Figure 6 
The genus level distribution of ileal and cecal microbiota among groups. 14IA: 14-d ileum control group; 14IB: 14-d ileum Astragalus group; 14IC: 14-d ileum L. Plantarum group; 14ID: 14-d ileum fermented Astragalus group; 14CA: 14-d cecum control group; 14CB: 14-d cecum Astragalus group; 14CC: 14-d cecum L. Plantarum group; 14CD: 14-d cecum fermented Astragalus group; 28IA: 28-d ileum control group; 28IB: 28-d ileum Astragalus group; 28IC: 28-d ileum L. Plantarum group; 28ID: 28-d ileum fermented Astragalus group; 28CA: 28-d cecum control group; 28CB: 28-d cecum Astragalus group; 28CC: 28-d cecum L. Plantarum group; 28CD: 28-d cecum fermented Astragalus group 